\title{
Genetic analysis, in silico prediction, and family segregation in long QT syndrome
}

\author{
Helena Riuró ${ }^{1,2}$, Oscar Campuzano ${ }^{1,2}$, Paola Berne ${ }^{3}$, Elena Arbelo ${ }^{3}$, Anna Iglesias ${ }^{1,2}$, Alexandra Pérez-Serra ${ }^{1}$, \\ Mònica Coll-Vidal ${ }^{1,4}$, Sara Partemi ${ }^{4}$, Irene Mademont-Soler ${ }^{1}$, Ferran Picó ${ }^{1}$, Catarina Allegue ${ }^{1,2}$, \\ Antonio Oliva ${ }^{4}$, Edward Gerstenfeld ${ }^{5}$, Georgia Sarquella-Brugada ${ }^{6}$, Víctor Castro-Urda $^{7}$, Ignacio Fernández-Lozano ${ }^{7}$, \\ Lluís Mont ${ }^{3}$, Josep Brugada ${ }^{3}$, Fabiana S Scornik ${ }^{1,2}$ and Ramon Brugada ${ }^{\star 1,2}$
}

The heritable cardiovascular disorder long QT syndrome (LQTS), characterized by prolongation of the QT interval on electrocardiogram, carries a high risk of sudden cardiac death. We sought to add new data to the existing knowledge of genetic mutations contributing to LQTS to both expand our understanding of its genetic basis and assess the value of genetic testing in clinical decision-making. Direct sequencing of the five major contributing genes, KCNQ1, KCNH2, SCN5A, KCNE1, and KCNE2, was performed in a cohort of 115 non-related LQTS patients. Pathogenicity of the variants was analyzed using family segregation, allele frequency from public databases, conservation analysis, and Condel and Provean in silico predictors. Phenotype-genotype correlations were analyzed statistically. Sequencing identified 36 previously described and 18 novel mutations. In $51.3 \%$ of the index cases, mutations were found, mostly in KCNQ1, KCNH2, and SCN5A; $5.2 \%$ of cases had multiple mutations. Pathogenicity analysis revealed 39 mutations as likely pathogenic, 12 as VUS, and 3 as non-pathogenic. Clinical analysis revealed that $75.6 \%$ of patients with QTc $\geq 500$ ms were genetically confirmed. Our results support the use of genetic testing of KCNQ1, KCNH2, and SCN5A as part of the diagnosis of LQTS and to help identify relatives at risk of SCD. Further, the genetic tools appear more valuable as disease severity increases. However, the identification of genetic variations in the clinical investigation of single patients using bioinformatic tools can produce erroneous conclusions regarding pathogenicity. Therefore segregation studies are key to determining causality.

European Journal of Human Genetics (2015) 23, 79-85; doi:10.1038/ejhg.2014.54; published online 26 March 2014

\section{INTRODUCTION}

Congenital long QT syndrome (LQTS), a cardiovascular disorder occurring in 1 in 2000 individuals $^{1}$ and is characterized on electrocardiogram by a QT interval prolongation that results from a repolarization abnormality within a structurally normal heart. ${ }^{2,3}$ The LQTS phenotype ranges from asymptomatic to the presence of syncopal episodes, seizures, malignant ventricular tachyarrhythmias (usually torsade de pointes), ventricular fibrillation, and sudden cardiac death (SCD). ${ }^{4,5}$

To date, mutations in five genes - KCNQ1 (KvLQT1 or Kv7.1, LQTS type 1$)^{6}$ and its associated $\beta$ subunit KCNE1 (mink, LQTS type 5); ${ }^{7}$ KCNH2 (hERG or Kv11.1, LQTS type 2$)^{8}$ and its associated $\beta$ subunit KCNE2 (mirp1, LQTS type 6$) ;{ }^{9}$ and SCN5A $\left(\mathrm{Na}_{\mathrm{v}} 1.5 \text {, LQTS type } 3\right)^{10}$ produce approximately $75 \%$ of LQTS cases. ${ }^{11-16}$ Although $90 \%$ of LQTS mutations are localized in these five genes, pathogenic mutations have been reported for 11 other genes: ANK2, KCNJ2, CACNA1C, CAV3, SCN4B, AKAP9, SNTA1, KCNJ5, CALM1, CALM $2,{ }^{17}$ and RYR $2 .{ }^{18}$

Recent guidelines recommend genetic investigation of arrhythmias for clinical purposes. ${ }^{19,20}$ Determining the genotype supports clinical diagnosis, improves treatment strategies, and may help identify risk of SCD..$^{20-22}$ In addition, positive genetic testing helps identify carrier family members who might also be at risk of SCD. ${ }^{20}$
However, as genetic studies unravel more disease associations, the use of genetic information in clinical decision-making becomes increasingly complex, especially when discerning pathogenic mutations from neutral variants and variants of uncertain significance (VUS). ${ }^{23-25}$

To examine the spectrum of pathogenic mutations and to generate new data toward assessing the value of genetic testing in clinical decision-making, we performed a genetic screening of the five recommended major LQTS-related genes from 115 non-related patients clinically diagnosed with LQTS.

\section{MATERIALS AND METHODS}

Ethics and approvals

This study was approved by the Ethics Committee of Hospital Josep Trueta (Girona, Spain) and conforms to the principles outlined in the Declaration of Helsinki.

\section{Sample population}

This study enrolled 115 non-related Caucasian LQTS patients. Clinical data, including 12-lead ECG, personal history of syncope, and family history, were collected. The QTc value was obtained from the 12-lead ECG based on the Bazzett's formula. The diagnosis of LQTS was assessed by the re-evaluated Schwartz diagnostic criteria., ${ }^{4}, 17 \mathrm{QTc} \geq 480 \mathrm{~ms}$ after exercise stress test was

${ }^{1}$ Cardiovascular Genetics Center, Institut d'Investigació Biomèdica de Girona, Girona, Spain; ${ }^{2}$ Department of Medical Sciences, Medical School, Universitat de Girona, Girona, Spain; ${ }^{3}$ Hospital Clínic de Barcelona, Barcelona, Spain; ${ }^{4}$ Institute of Forensic Medicine, Catholic University, Rome, Italy; ${ }^{5}$ UCSF School of Medicine, San Francisco, CA, USA; ${ }^{6}$ Hospital Sant Joan de Déu, Barcelona, Spain; ${ }^{7}$ Hospital Puerta de Hierro, Madrid, Spain

*Correspondence: Professor R Brugada, School of Medicine, Universitat de Girona; Cardiovascular Genetics Center, Institut d'Investigació Biomèdica de Girona, C/ Pic de Peguera 11, Girona 17003, Spain. Tel: +34 972 183366; Fax: +34 972 183367; E-mail: ramon@brugada.org

Received 4 October 2013; revised 23 January 2014; accepted 19 February 2014; published online 26 March 2014 
counted as 1 point. ${ }^{17}$ Deafness was not reported in any of the patients analyzed.

All individuals signed a written informed consent to participate in the study. Informed consent of all patients was obtained in accordance with the Review Board guidelines of the Hospital Josep Trueta and Universitat of Girona (Girona, Spain).

\section{Genetic analysis}

Total genomic DNA was isolated from blood samples using the Puregene DNA purification Kit (Gentra System, Minneapolis, MI, USA). Exons and exonintron boundaries of these genes were amplified (Verities PCR, Applied Biosystems, Austin, TX, USA). The PCR products were purified with Exosap-IT (USB Corporation, Cleveland, OH, USA) and directly sequenced in both directions (BigDye Terminator v3.1 Cycle Sequencing Kit, 3130XL Genetic Analyser, Applied Biosystems). Obtained DNA sequences were compared with the reference sequence of KCNQ1 (NM_000218.2; MIM\# 607542), KCNH2 (NM_000238.3; MIM\# 152427), SCN5A (NM_198056.2; MIM\# 600163), KCNE1 (NM_000219.3; MIM\# 176261), and KCNE2 (NM_172201.1; MIM\# 603796) using the SeqScape v2.6 software (Applied Biosystems). Gene symbols were verified with HUGO Gene Nomenclature Committee (http://www.genenames.org/), and all GenBank reference sequences were obtained from the Consensus CDS Protein Set Database (http:// www.ncbi.nlm.nih.gov/CCDS/).

Genetic variations were contrasted with HGMD Professional 2013.4 (http:// www.biobase-international.com/), 1000 genomes project browser (http:// browser.1000genomes.org), and dbSNP database v.137 (http://www.ncbi.nlm. nih.gov/projects/SNP). Exome Variant Server-NHLBI Exome Sequencing Project (ESP6500SI-V2, http://snp.gs.washington.edu/EVS/) and dbSNP database were used to find minor allele frequencies (MAFs) in all populations. UniProt database (http://www.uniprot.org/) was used to analyze the conservation of affected amino-acid regions between species. Mutation pathogenicity was predicted through two in silico programs: Condel (CONsensus DELeteriousness score of non-synonymous single-nucleotide variants), ${ }^{26}$ which integrates the outputs of three tools: SIFT, Polyphen2, and Mutationassessor; and Provean v.1.1 (Protein Variation Effect Analyzer), ${ }^{27}$ which is the first predictor that includes in-frame insertions/deletions.

Nomenclature is cited according to the HGVS guidelines (www.hgvs.org/ mutnomen): 'c.' indicates a coding DNA sequence and 'p.' indicates a protein sequence. Sequence variants were checked using the Mutalyzer program (http://www.LOVD.nl/mutalyzer).
Sequence changes altering coding regions were defined as mutations, and only exonic mutations were included in the study. Mutations with an MAF in all populations $<1 \%$ were considered as presuming disease alleles. Mutations with an $\mathrm{MAF} \geq 1 \%$ were excluded from the study. Novel mutations identified have been submitted to the LOVD v.3.0 (Leiden Open Variation Database, http://www.lovd.nl/3.0/home).

\section{Statistical analysis}

Statistical analyses were performed using the IBM SPSS Statistics 21 (IBM Corp., Armonk, NY, USA). Mean values are expressed as mean \pm SD. Statistical comparisons were performed using the unpaired Student's $t$-test and chisquared test. Results were considered statistically significant when $P<0.05$.

\section{RESULTS}

In our cohort of 115 LQTS-affected non-related individuals (mean age of diagnosis $33.9 \pm 19$ years, $62.5 \%$ females), the mean QTc was $489.1 \pm 56.5 \mathrm{~ms}$. Syncope occurred in $57.1 \%$ of our patients, SCD had episodes occurred in $16.7 \%$, and previous family history of LQTS and/or SCD was noted in 55.4\% (Table 1).

\section{Mutation analysis}

We identified 59 index cases $(51.3 \%)$ with a mutation in at least one of the 5 analyzed genes and 56 patients without mutations in these genes $(48.7 \%)$ (Table 1). Genes most frequently found to have mutations were $\mathrm{KCNH} 2$ (17.4\% of total patients), KCNQ1 (14.8\%) and SCN5A (9.6\%); and presence of more than one mutation in the same or multiple genes was identified in six index cases $(5.2 \%)$ (Figure 1a). In total, 54 different mutations were found, 18 were novel, and 10 previously described mutations were identified in $>1$ patient (Supplementary Tables S1 and S2).

Pathogenicity of novel mutations. None of the 18 novel mutations were present in the general population. Alignment analysis revealed that only KCNH2 p.(Gly238Arg) was not conserved between species (Supplementary Figure S1).

Segregation analysis from the eight available families carrying novel mutations (Table 2) revealed that two families segregated with the clinical diagnosis, five families showed incomplete penetrance (IP),

Table 1 Clinical data from non-related LQTS patients

\begin{tabular}{|c|c|c|c|c|c|c|c|}
\hline & $\begin{array}{c}\text { Number of } \\
\text { patients }\end{array}$ & Mean age (years) & Gender (female) & Syncope (yes) & Previous SCD (yes) & Family history (yes) & Mean QTC (ms) \\
\hline Total & 115 & $33.9 \pm 19$ (109) & $62.5 \%(112)$ & $57.1 \%(105)$ & $16.7 \%(102)$ & $55.4 \%(101)$ & $489.1 \pm 56.5$ (97) \\
\hline Non-carriers & 56 & $38.9 \pm 19.1^{\mathrm{a}}(51)$ & $58.5 \%(53)$ & $59.2 \%(49)$ & $16.3 \%(49)$ & $56.3 \%(48)$ & $470.8 \pm 48.1^{a}(44)$ \\
\hline \multicolumn{8}{|l|}{ Carriers } \\
\hline Total & 59 & $29.6 \pm 17.9(58)$ & $66.1 \%(59)$ & $55.4 \%(56)$ & $17.0 \%(53)$ & $54.7 \%(53)$ & $504.2 \pm 58.8(53)$ \\
\hline KCNQ1 mutation & 17 & $24.5 \pm 15.0(14)$ & $70.6 \%(17)$ & $64.3 \%(14)$ & $14.3 \%(14)$ & $73.3 \%(15)$ & $514.4 \pm 59.1$ \\
\hline $\mathrm{KCNH} 2$ mutation & 20 & $29.4 \pm 14.6(18)$ & $70 \%(20)$ & $70 \%(20)$ & $16.7 \%(18)$ & $52.9 \%(17)$ & $500 \pm 60.7(18)$ \\
\hline SCN5A mutation & 11 & $39.0 \pm 19.1$ (9) & $63.6 \%(11)$ & $27.3 \%^{\mathrm{b}}(11)$ & $20 \%(10)$ & $40 \%(10)$ & $492.2 \pm 60.1(9)$ \\
\hline KCNE1 mutation & 3 & $29.3 \pm 30.9(3)$ & $33.3 \%(3)$ & $66.7 \%(3)$ & $0.0 \%(3)$ & $0 \%(3)$ & $470.7 \pm 17.9(3)$ \\
\hline KCNE2 mutation & 2 & $35.0 \pm 1.41(2)$ & $50 \%(2)$ & $50 \%(2)$ & $50 \%(2)$ & $50 \%(2)$ & $467.5 \pm 38.9(2)$ \\
\hline Single mutation & 53 & $29.8 \pm 17.0(52)$ & $66.0 \%(53)$ & $58.0 \%(50)$ & $17.0 \%(47)$ & $53.2 \%(47)$ & $503.4 \pm 62.4(46)$ \\
\hline Multiple mutations & 6 & $28.2 \pm 26.5(6)$ & $66.7 \%(6)$ & $33.3 \%(6)$ & $16.7 \%(6)$ & $66.7 \%(6)$ & $510.5 \pm 9.9(6)$ \\
\hline Non-pathogenic mutation & 5 & $35.4 \pm 19.9(5)$ & $60.0 \%(5)$ & $60.0 \%(5)$ & $40.0 \%(5)$ & $40.0 \%(5)$ & $473.0 \pm 26.8(5)$ \\
\hline VUS mutation & 7 & $35.0 \pm 21.8(7)$ & $42.9 \%(5)$ & $57.1 \%(7)$ & $0.0 \%(6)$ & $42.9 \%$ & $453.2 \pm 37.4(6)^{c}$ \\
\hline Pathogenic mutation & 41 & $28.2 \pm 15.9(40)$ & $73.2 \%(41)$ & $57.9 \%(38)$ & $16.7 \%(36)$ & $57.1 \%(35)$ & $515.9 \pm 64.1(36)$ \\
\hline
\end{tabular}

${ }^{a}$ Non-carriers vs carriers: $P<0.01$.

${ }^{\mathrm{b}}$ Carriers with $S C N 5 \mathrm{~A}$ mutation vs carriers with $K C N H 2$ mutation: $P<0.05$.

cVUS carriers vs pathogenic mutations carriers: $P<0.05$.

Values are presented as mean \pm SD. Complete clinical information was not available for each patient; thus the number of patients used to obtain the percentage or mean values are specified in parentheses. 
a

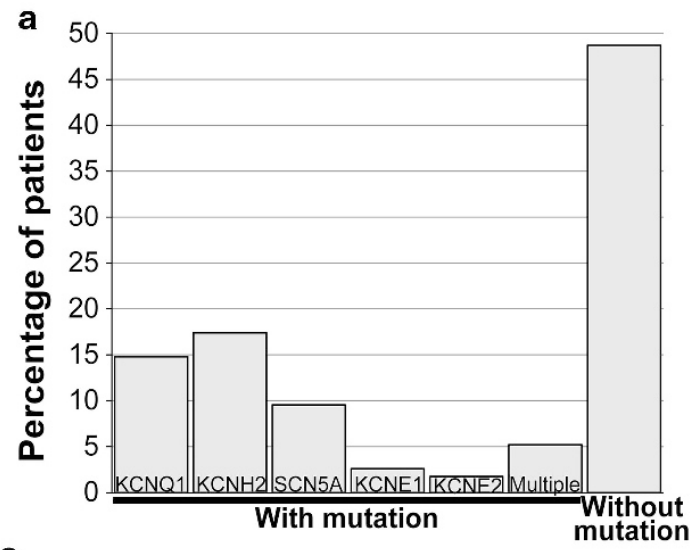

b



C

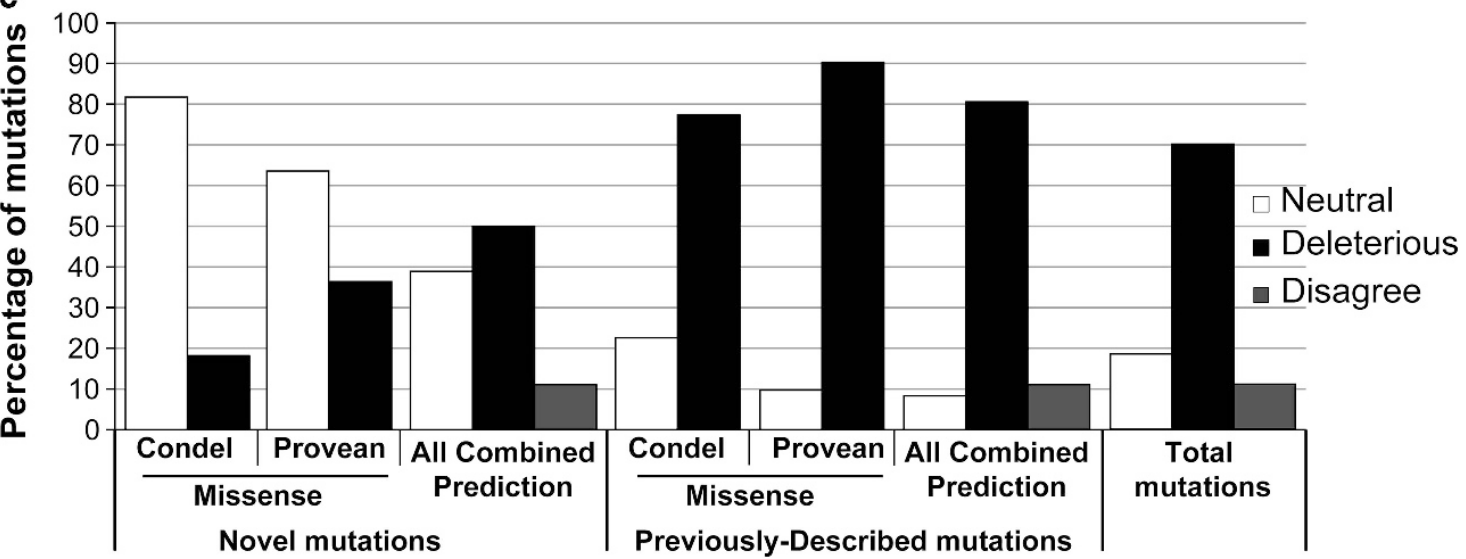

Figure 1 Mutation distribution in non-related LQTS patients. (a) Distribution of all mutations in our non-related LQTS cohort $(n=115)$. Bars represent the percentages of patients with mutations in KCNQ1,KCNH2, SCN5A, KCNE1, and KCNE2 or with multiple mutations ( $\geq 2$ mutations in $\geq 1$ genes) and without mutations in the five analyzed genes. (b) Distribution of radical mutations (in-frame deletions, frameshift deletions, and nonsense mutations; dark gray bars) and missense mutations (light gray bars) of the novel mutations $(n=18)$ and previously described mutations $(n=36)$ for the 54 mutations identified from 59 patients. (c) Distribution of in silico predictions for novel missense mutations $(n=11)$ and previously described missense mutations $(n=31)$. Mutations were classified as neutral (white bars) or deleterious (black bars). In combined prediction, bars represent radical (considered deleterious) and missense mutations predicted from both novel $(n=18)$ and previously described $(n=36)$ mutations. Disagreement bars represent the mutations with inconclusive data due to a different prediction between the two used tools (gray bars). Total mutations are the combined prediction for both novel and previously described radical and missense mutations.

and one mutation did not segregate with the clinical data. However, four index cases with novel mutations with IP also harbored previously described mutations in the same or other genes (see 'Pathogenicity of multiple mutations' paragraph and Figure 2).

We identified 7 radical mutations (in-frame deletion, frameshift deletion, and nonsense novel mutations) and 11 missense mutations (Table 2 and Figure 1b). Nonsense and frameshift mutations were considered deleterious, as in the literature, ${ }^{28}$ because no in silico pathogenicity predictors exist for them. In-frame deletions were predicted as deleterious by Provean tool. In silico pathogenicity prediction from the 11 novel missense mutations revealed that 2 mutations had a deleterious effect; 3 mutations localized in the $\mathrm{KCNH} 2 \mathrm{C}$-terminus, 2 were predicted as neutral but predictions for the third were discordant; and 4 of the 5 mutations in the $\mathrm{KCNH} 2$ $\mathrm{N}$-terminus or SCN5A IDL were predicted as neutral, the fifth had discordant predictions (Figure 1c). Nevertheless, none of these variants were found in the general population, and alignment analysis revealed all but one (KCNH2 p.(Gly238Arg)) as conserved amino acids.

Pathogenicity of previously described mutations. Of the known mutations confirmed in our population, 5 were radical mutations and 31 were missense mutations (Figure 1b). Three mutations occurred in the general population with an $\mathrm{MAF}<1 \%$ (Supplementary Table S2). Alignment analysis showed conservation of all but three of the affected amino acids. Family members were available for 19 index cases. Segregation analysis revealed that 10 mutations segregated with the disease, 6 segregated with IP, 1 did not segregate, and 2 were de novo (Supplementary Tables S1 and S2).

Based on these analyses and other reports, we considered 25 previously described mutations as probably pathogenic mutations (radical mutations or missense mutations without frequency in the general population, amino-acid conservation, and positive segregation from the families available) (Supplementary Table S1). Our in silico analysis predicted 23 out of the 25 probably pathogenic mutations as deleterious, and 2 mutations were predicted differently between the tools used (Figure 1c and Supplementary Table S1).

In addition, we considered four previously described mutations as having uncertain significance (Supplementary Table S2): amino acids KCNQ1 p.Pro73 and SCN5A p.Pro1008 were not conserved between species. Further, KCNQ1 p.(Pro73Thr) was previously described as a VUS, ${ }^{28}$ and SCN5A p.(Pro1008Ser) had been associated with other arrhythmogenic diseases, similar to SCN5A p.(Arg620Cys). ${ }^{29,30}$ Accordingly, in silico programs predicted these three mutations as neutral (Supplementary Table S2). In contrast, although SCN5A 


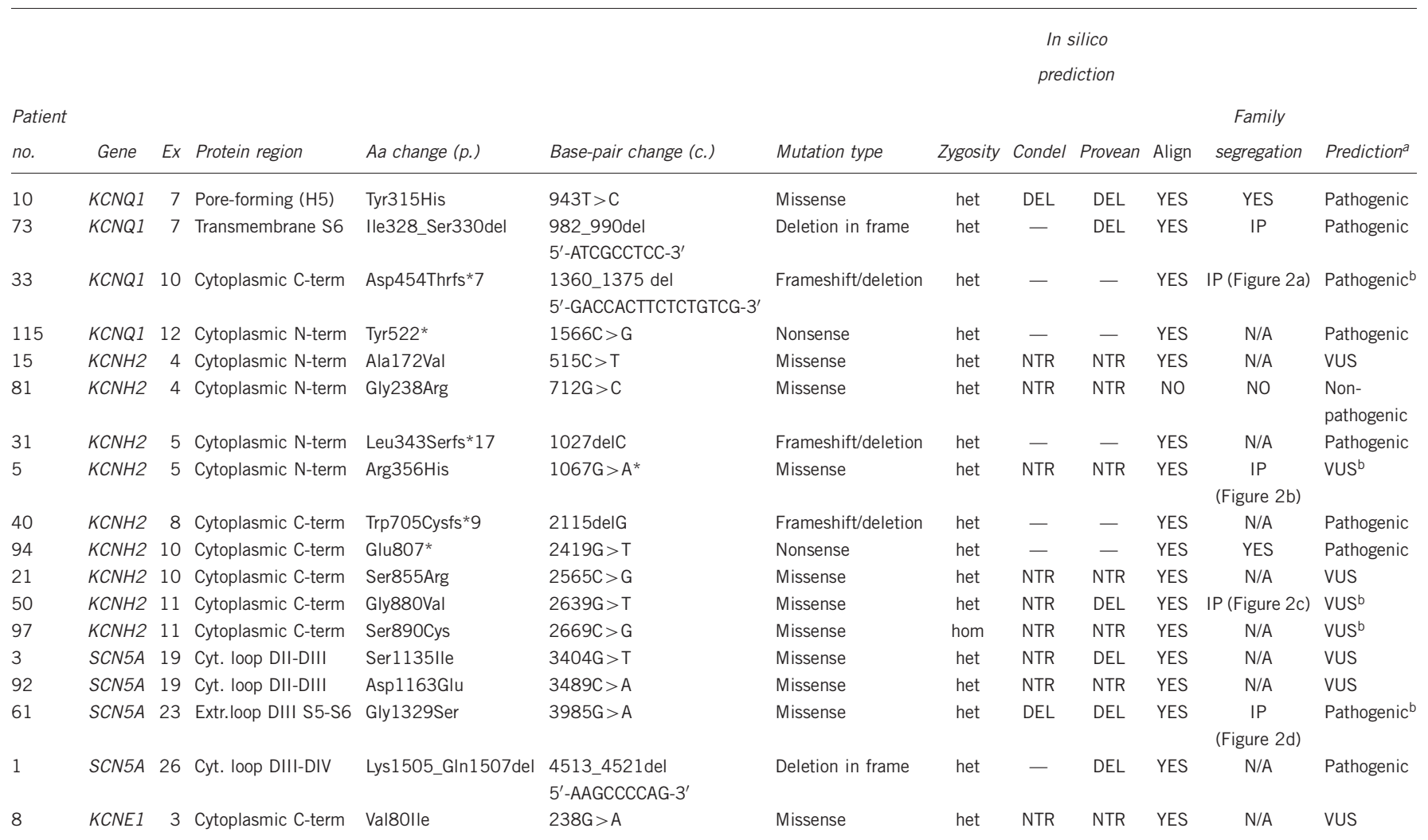

Abbreviations: Aa, amino-acid; Cyt., cytoplasmatic; Ex, exon; Extr., extracellular; het, heterozygous genotype; hom, homozygous genotype.

a Our prediction after the analysis of the results obtained from segregation analysis, amino acid sequence alignment, and in silico tools. The prediction was in terms of probability.

'The novel mutation was found together with other mutations in the family.

Pathogenic predictions were obtained from Condel and Provean (see Materials and Methods). Frameshift/deletions and nonsense mutations could not be predicted by either of the predictor programs; we assumed that these types of mutations are deleterious. In-frame deletions could only be predicted by Provean program. We described the pathogenicity with the same scores as given by the programs: NTR, neutral; DEL, deleterious; N/A, not available; IP, incomplete penetrance.

p. (Ser216Leu) was found in the general population $(\mathrm{MAF}=0.1 \%)$ and was described as a VUS, ${ }^{24}$ our in silico analysis predicted it as deleterious (Supplementary Table S2).

Finally, we considered two mutations as potentially non-causative mutations: we found KCNE1 p.(Asp85Asn) in three non-related index cases; in one case, this mutation occurred with other mutations (Figure 2d and Supplementary Table S2). Although this mutation is found in $<1 \%$ of the general population, it has been described as an LQTS modulator ${ }^{31,32}$ and associated with a QT prolongation. ${ }^{33}$ Similarly, KCNE2 p.(Thr8Ala) was found in $0.5 \%$ of the general population but was also described as a VUS. ${ }^{24}$ We found this variant in two unrelated index cases; segregation analysis from one of the index cases revealed that the mutation did not segregate with the disease, excluding it as the causal mutation (Supplementary Table S2). The in silico tools did not agree in the pathogenicity of those mutations.

Pathogenicity of multiple mutations. We found six index cases with multiple mutations; segregation was assessed for five of these cases (no family information was available for patient no. 97) (Figure 2). Three families had two mutations, which occurred in the same gene in two families (Figure $2 \mathrm{~b}$ and $\mathrm{c}$ ) and on a different gene in one (Figure 2e); two families had three mutations, and in both families one of the mutations was a VUS or a modulator variant (Figures 2a and $\mathrm{d}$ ).

The index cases of families a, c, and d, carrying multiple mutations, showed a more severe phenotype within their own family: In family a the index case (II.2, Figure 2a) had LQTS, which was diagnosed at the age of 8 years; in family $c$ the index case (III.1, Figure 2c) was the only family member with LQTS; and in family $d$ the index case (IV.3, Figure 2d) was the only family member with LQTS, diagnosed after an aborted SCD. In contrast, the index case in family b (II.1, Figure $2 \mathrm{~b}$ ), who was the only member harboring the two mutations, had no symptoms until she was 74 years old (Figure $2 \mathrm{~b}$ ). Finally, the three sisters from family e carried the two mutations and presented prolonged QT interval and previous syncope (Figure 2e).

\section{Clinical data analysis}

Differences in the clinical phenotype between carrier or non-carrier patients. Of all the genetic carriers, $66.1 \%$ were females, and significant differences were observed in mean QTc value and age of diagnosis between patients with or without an identified mutation $(470.8 \pm 48.1 \mathrm{~ms}$ and $38.9 \pm 19.1$ years; and $504 \pm 58.8 \mathrm{~ms}$ and $29.6 \pm 17.9$ years, respectively). In contrast, there were no significant differences in the remaining severity predictors between genotypically positive and negative patients. Multiple mutations were present in $5.2 \%$ of the index cases. No differences were detected in the clinical data between patients with single or multiple mutations (Table 1).

Differences in the clinical phenotype depending on mutation pathogenicity. We compared clinical data from patients with VUS mutations and from those with probably pathogenic mutations. Indeed, QTc was significantly longer in patients with pathogenic mutations than in 
a

KCNQ1 p.Pro73Thr KCNQ1 p.Gly168Arg KCNQ1 p.Asp454Thrfs* ${ }^{\dagger}{ }^{\dagger}$

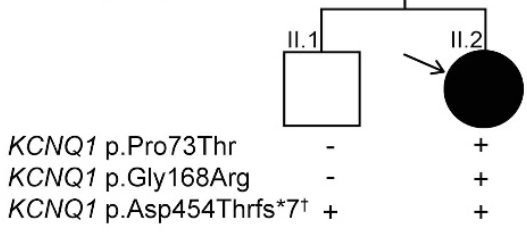

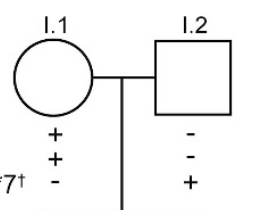

b<smiles>c1ccc(-c2cccnc2)cc1</smiles>

KCNH2 p.Arg356His ${ }^{\dagger}$ KCNH2 p.Ala561Val

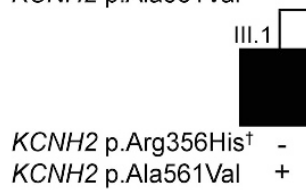

KCNH2 p.Arg $356 \mathrm{His}^{\dagger}$ KCNH2 p.Ala561Val

c

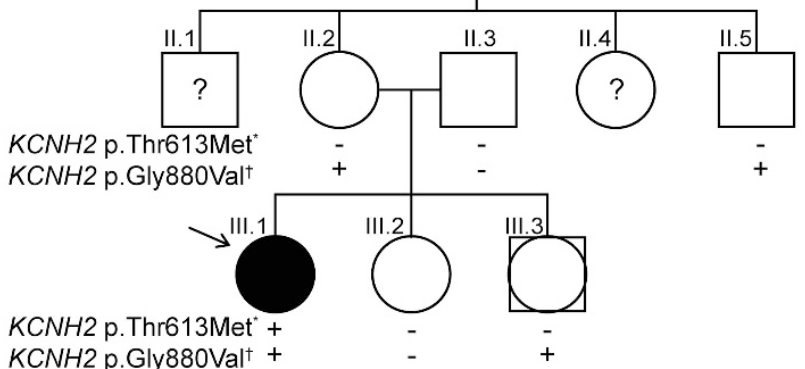
KCNH2 p.Gly880 $\mathrm{Val}^{\dagger}+$

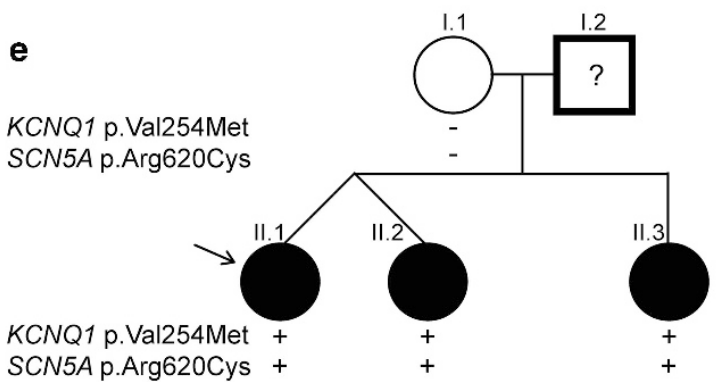

Figure 2 Familial segregation analysis from patients with multiple mutations. Families (a-e): Arrows indicate the index cases. Circles represent female, and squares represent male; circle-within-squares represent unknown sex. Clinically affected individuals are shown in black. Symbols with a thick black line represent symptomatic carriers without LQTS at the time of the study but susceptible of exhibiting the disorder. The + symbol represents mutation carriers and-symbol the non-carriers from the different mutations found in the index case. Interrogation marks (?) represent family members for whom genetic information was not available. Asterisk (*) signifies de novo mutations; and † signifies novel mutations.

patients with VUS $(515.9 \pm 64.1$ and $453.2 \pm 37.4 \mathrm{~ms}$, respectively; Table 1).

To better understand this finding, we assessed the prevalence of mutation identification according to Schwartz Score (SS) (Figure 3). The percentage of patients with a genetic diagnosis increased to $63.6 \%$ in $S S \geq 3.5$. In addition, the percentage rose to $75.7 \%$ when we limited to $\mathrm{QTc} \geq 500 \mathrm{~ms}$. In this patient population ( $\mathrm{SS} \geq 3.5$ and QTc $\geq 500 \mathrm{~ms}$ ), the presence of probably pathogenic mutations was higher and the number of VUS mutations was lower compared with patients with $\mathrm{SS}<3.5$ and QTc $<500 \mathrm{~ms}$ (Figure 3).
Phenotype related to mutated gene. We analyzed clinical data to identify phenotypic differences associated with variants of the five genes (Table 1). The mean age of diagnosis and QTc value were not influenced by the analyzed genes. However, we found that patients with a mutation in SCN5A were significantly less likely to have previous syncope compared with patients carrying mutations in KCNH2 (27.3 and 70\%, respectively).

Phenotype related to mutation type, protein region, and zygosity. Missense mutations, heterozygous genotype, and mutations in the C-terminal 


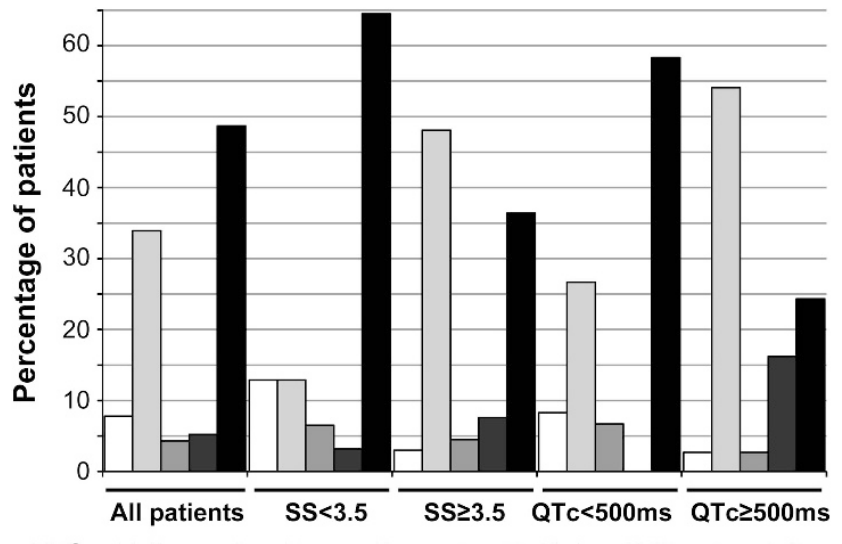

$\square$ VUS $\square$ Pathogenic $₫$ Non-pathogenic $₫$ Multiple - Without mutation

Figure 3 Mutation pathogenicity yield correlates with LQTS prolongation. Bars represent the percentages determined for all patients $(n=115)$, patients with Schwartz Score $(\mathrm{SS})<3.5 \quad(n=31), \quad \mathrm{SS} \geq 3.5 \quad(n=66)$, QTc $<500 \mathrm{~ms}(n=60)$, or QTc $\geq 500 \mathrm{~ms}(n=37)$ for different mutation groups according to their pathogenicity: VUS, probably pathogenic, probably non-pathogenic, multiple variations, and non-carriers.

domain were the most common identified (Supplementary Table S3). No significant differences were seen when we evaluated whether the mutation characteristics influenced the risk factors or the severity of clinical characteristics (Supplementary Table S3).

\section{DISCUSSION}

In our cohort of patients with LQTS, the mean age of diagnosis and the mean QTc values were similar to previous studies. ${ }^{12-14,16}$ In addition, also similar to a recent report, ${ }^{34}$ we found a ratio near to $2: 1$ between women and men. Thus our population appears to be a representative sampling of Caucasian LQTS patients.

We identified mutations in the five most prevalent disease-related genes in $51.3 \%$ of the LQTS-affected patients. Previous studies indicated that $70-75 \%$ of patients carry a mutation in one of these five genes, ${ }^{11-13}$ but recent studies have reported a similar genetic finding in close to $50 \%$ of LQTS patients. ${ }^{14,16}$ The percentage increased to $75 \%$ when patients had a SS $\geq 4 .{ }^{13}$ According to the modified risk scores, ${ }^{17}$ we analyzed patients with $S S \geq 3.5$. Our percentage of patients with an identified mutation rose to $63.6 \%$, increasing to $75.7 \%$ when we analyzed only patients with QTc $\geq 500 \mathrm{~ms}$. Importantly, the likelihood of finding a probably pathogenic mutation increased in both cases.

The number of patients with identified mutations increases in younger patients. ${ }^{12,13,16}$ Our population age ranged from birth to 77 years old. However, if we analyzed patients within an age range of diagnosis from birth to $\leq 40$ years old, the percentage of patients with an identified mutation increased to $62.3 \%$.

Therefore, in agreement with previous studies, ${ }^{13,14}$ our data indicate the genetic diagnosis of LQTS syndrome is more effective in more severe cases, in younger patients, and in those with higher QTc values.

Although we found significant differences in the occurrence of syncope between patients carrying mutations in SCN5A and $\mathrm{KCNH} 2$, in contrast to other studies we did not see differences in life-threatening cardiac events between patients carrying mutations in KCNQ1, KCNH2, and SCN5A.22,35 The size of the cohort may have precluded a conclusive genotype-phenotype analysis.

\section{Pathogenicity prediction with in silico tools}

With the use of genetics as a diagnostic tool, often performed solely in the index case without segregation analysis, the challenge to define true pathogenic association is tremendous..$^{23,25,28}$ However, new in silico tools are helping define the pathogenicity of variants. Here, we used two such programs: Condel (including Polyphen2, SIFT, and Mutation assessor) and Provean (including prediction of in-frame deletions and insertions). Based on literature, MAF, segregation, and conservation data, we considered 25 previously described mutations as probably pathogenic. Condel and Provean predicted as deleterious 92 and $100 \%$ of them, respectively. This accuracy is even higher than previously claimed with those predictors, believed to be near $80 \% .^{26,27,36}$

Of all the mutations, three were considered non-causative from segregation analysis, conservation, and previous studies. Condel predicted all of them as neutral. In contrast, Provean predicted two of them as deleterious. These results are in accordance with the nearly $20 \%$ false-positive rates expected from these programs. ${ }^{36}$

In silico pathogenicity prediction of the 11 novel missense mutations revealed that 2 novel mutations had a deleterious effect, according to the high probability of pathogenicity for the region (pore-forming region and the loop DIII S5-S6), ${ }^{23}$ amino-acid conservation, and family segregation. However, the in silico predictions obtained for novel mutations in protein areas with lower pathogenicity probability $(50-80 \%$ probability in C-terminal mutations $v s<50 \%$ in $\mathrm{N}$-terminal or interdomain loop mutations (IDL) in $S C N 5 A)^{23}$ were more ambiguous. Moreover, from our analysis, Condel and Provean differed in 6 out of the 54 mutations evaluated $(11 \%)$. Some studies have shown discordance between Polyphen 2 and SIFT in silico prediction programs to be more frequent than the accordance on their predictions. ${ }^{28}$ Although in our analysis the percentage of discordance was low, segregation analysis and/or functional studies are necessary to verify the pathogenicity or neutrality of these mutations. ${ }^{28,37}$ Taking together, without validation, some of these mutations predicted as neutral or discordance will remain of uncertain significance, confounding genetic diagnosis.

\section{Segregation analysis: key to validating the predictions}

In several different situations, segregation analysis is critical to understanding the contribution of a mutation to LQTS. First, we identified mutations associated with Brugada syndrome (SCN5A p. $(\text { Arg620Cys) })^{29}$ and cardiac conduction disease (SCN5A p.(Pro1008Ser) $),{ }^{30}$ which result from sodium channel defects. The discovery of mutations that are associated with different arrhythmogenic diseases could be consistent with the overlapping syndromes seen in these sodium channel diseases. ${ }^{38,39}$ However, the contribution of these mutations remain unclear; thus their presence makes diagnosis, and especially medical therapy, difficult. Therefore, additional familial evaluation is critical for further medical decisions.

Additionally, five of the identified mutations were segregating with other mutations. Segregation analysis suggested that some mutations with uncertain effects could modulate the phenotype when occurring with other mutations (KCNQ1 p.(Pro73Thr) in Family a, KCNH2 p.(Arg356His) in Family b, and KCNH2 p.(Gly880Val) in Family c).

Finally, nine novel missense mutations had neutral or discordant predictions, but only one, KCNH2 p.(Gly238Arg), exhibited a lack of segregation that excluded it as the main cause of the disease in the family. The rest of them were considered VUS. Therefore, in these situations, prediction only is not sufficient for understanding the contribution of variants to disease. 


\section{CONCLUSIONS}

Our data support that in silico predictors, in general, are useful for determining pathogenicity. However, prediction may be uncertain or inaccurate in $\sim 22 \%$ of cases. In our cohort of LQTS, with a rate of identified mutations near $50 \%$, such uncertainty is not to be taken lightly, especially considering that the genes of interest that have been widely studied for their clear association with pathology. Thus, in silico predictors should be combined with other tools and family segregation analyses to verify pathogenicity. ${ }^{25}$

In this era of rapid genetic sequencing, the lesson from these studies is twofold. First, we are not yet ready to use genetic testing as a diagnostic tool in the least-studied genes. The lack of robust genetic associations with these genes will still hamper clinical decisionmaking. Second, segregation studies and family investigation continue to be critical in any pathogenicity study. If the genotype does not fit the family phenotype, no matter what, genetic causality cannot be proven. This has important implications in genetic diagnosis, in which the analysis is often performed solely in the index patient, without family investigation.

\section{CONFLICT OF INTEREST}

$\mathrm{RB}$ is a consultant for Ferrer Incode. The other authors declare no conflict of interest.

\section{ACKNOWLEDGEMENTS}

HR is a recipient of the PFIS predoctoral fellowship III (FI09/00336) and OC is a recipient of a Sara Borrell postdoctoral fellowship (CD09/00055) from the Instituto de Salud Carlos III. This work was supported by CNIC-Translational 2008 (CNIC-03-2008), the Spanish Ministry of Health (Red Cooperativa de Insuficiencia Cardiaca (REDINSCOR) RD06/03/0018), and Obra Social 'la Caixa'

1 Schwartz PJ, Stramba-Badiale M, Crotti L et al: Prevalence of the congenital long-QT syndrome. Circulation 2009; 120: 1761-1767.

2 Crotti L, Celano G, Dagradi F, Schwartz PJ: Congenital long QT syndrome. Orphanet J Rare Dis 2008; 3: 18.

3 Goldenberg I, Moss AJ: Long QT syndrome. J Am Coll Cardiol 2008; 51: 2291-2300.

4 Schwartz PJ, Moss AJ, Vincent GM, Crampton RS: Diagnostic criteria for the long QT syndrome. An update. Circulation 1993; 88: 782-784.

5 Roden DM: Clinical practice. Long-QT syndrome. N Engl J Med 2008; 358 : 169-176.

6 Wang Q, Curran ME, Splawski I et al: Positional cloning of a novel potassium channe gene: KVLQT1 mutations cause cardiac arrhythmias. Nat Genet 1996; 12: 17-23.

7 Splawski I, Tristani-Firouzi M, Lehmann MH, Sanguinetti MC, Keating MT: Mutations in the hminK gene cause long QT syndrome and suppress IKs function. Nat Genet 1997; 17: 338-340.

8 Curran ME, Splawski I, Timothy KW, Vincent GM, Green ED, Keating MT: A molecular basis for cardiac arrhythmia: HERG mutations cause long QT syndrome. Cell 1995; 80: 795-803.

9 Abbott GW, Sesti F, Splawski I et al: MiRP1 forms IKr potassium channels with HERG and is associated with cardiac arrhythmia. Cell 1999; 97: 175-187.

10 Wang Q, Shen J, Splawski I et al: SCN5A mutations associated with an inherited cardiac arrhythmia, long QT syndrome. Cell 1995; 80: 805-811.

11 Napolitano C, Priori SG, Schwartz PJ et al: Genetic testing in the long QT syndrome: development and validation of an efficient approach to genotyping in clinical practice. JAMA 2005: 294: 2975-2980.

12 Splawski I, Shen J, Timothy KW et al: Spectrum of mutations in long-QT syndrome genes. KVLQT1, HERG, SCN5A, KCNE1, and KCNE2. Circulation 2000; 102: $1178-1185$

13 Tester DJ, Will ML, Haglund CM, Ackerman MJ: Compendium of cardiac channel mutations in 541 consecutive unrelated patients referred for long QT syndrome genetic testing. Heart Rhythm 2005; 2: 507-517.
14 Kapplinger JD, Tester DJ, Salisbury BA et al: Spectrum and prevalence of mutations from the first 2500 consecutive unrelated patients referred for the FAMILION Iong QT syndrome genetic test. Heart Rhythm 2009; 6: 1297-1303.

15 Hedley PL, Jorgensen P, Schlamowitz S et al: The genetic basis of long QT and short QT syndromes: a mutation update. Hum Mutat 2009; 30: 1486-1511.

16 Stattin EL, Bostrom IM, Winbo A et al: Founder mutations characterise the mutation panorama in 200 Swedish index cases referred for Long QT syndrome genetic testing. BMC Cardiovasc Disord 2012; 12: 95

17 Schwartz PJ, Ackerman MJ, George Jr AL, Wilde AA: Impact of genetics on the clinical management of channelopathies. J Am Coll Cardiol 2013: 62: 169-180.

18 Kauferstein S, Kiehne N, Erkapic D et al: A novel mutation in the cardiac ryanodine receptor gene (RyR2) in a patient with an unequivocal LQTS. Int J Cardiol 2011; 146: 249-250.

19 Zipes DP, Camm AJ, Borggrefe $M$ et al: ACC/AHA/ESC 2006 Guidelines for Management of Patients With Ventricular Arrhythmias and the Prevention of Sudden Cardiac Death: a report of the American College of Cardiology/American Heart Association Task Force and the European Society of Cardiology Committee for Practice Guidelines (writing committee to develop Guidelines for Management of Patients With Ventricular Arrhythmias and the Prevention of Sudden Cardiac Death): developed in collaboration with the European Heart Rhythm Association and the Heart Rhythm Society. Circulation 2006; 114: e385-e484.

20 Gollob MH, Blier L, Brugada R et al: Recommendations for the use of genetic testing in the clinical evaluation of inherited cardiac arrhythmias associated with sudden cardiac death: Canadian Cardiovascular Society/Canadian Heart Rhythm Society joint position paper. Can J Cardiol 2011; 27: 232-245.

21 Moss AJ, Zareba W, Kaufman ES et al: Increased risk of arrhythmic events in long-QT syndrome with mutations in the pore region of the human ether-a-go-go-related gene potassium channel. Circulation 2002; 105: 794-799.

22 Priori SG, Schwartz PJ, Napolitano C et al: Risk stratification in the long-QT syndrome. N Engl J Med 2003; 348: 1866-1874.

23 Kapa S, Tester DJ, Salisbury BA et al: Genetic testing for long-QT syndrome: distinguishing pathogenic mutations from benign variants. Circulation 2009; 120: 1752-1760.

24 Refsgaard L, Holst AG, Sadjadieh G, Haunso S, Nielsen JB, Olesen MS: High prevalence of genetic variants previously associated with LQT syndrome in new exome data. Eur J Hum Genet 2012; 20: 905-908.

25 Giudicessi JR, Ackerman MJ: Genetic testing in heritable cardiac arrhythmia syndromes: differentiating pathogenic mutations from background genetic noise. Curr Opin Cardiol 2013; 28: 63-71.

26 Gonzalez-Perez A, Lopez-Bigas N: Improving the assessment of the outcome of nonsynonymous SNVs with a consensus deleteriousness score, Condel. Am J Hum Genet 2011; 88: 440-449.

27 Choi Y, Sims GE, Murphy S, Miller JR, Chan AP: Predicting the functional effect of amino acid substitutions and indels. PLoS One 2012; 7: e46688.

28 Giudicessi JR, Kapplinger JD, Tester DJ et al: Phylogenetic and physicochemical analyses enhance the classification of rare nonsynonymous single nucleotide variants in type 1 and 2 long-QT syndrome. Circ Cardiovasc Genet 2012; 5: 519-528.

29 Kapplinger JD, Tester DJ, Alders $M$ et al: An international compendium of mutations in the SCN5A-encoded cardiac sodium channel in patients referred for Brugada syndrome genetic testing. Heart Rhythm 2010; 7: 33-46.

$30 \mathrm{Hu} \mathrm{D}$, Barajas-Martinez H, Nesterenko VV et al: Dual variation in SCN5A and CACNB2b underlies the development of cardiac conduction disease without Brugada syndrome. Pacing Clin Electrophysiol 2010; 33: 274-285.

31 Nishio $\mathrm{Y}$, Makiyama T, Itoh H et al: D85N, a KCNE1 polymorphism, is a diseasecausing gene variant in long QT syndrome. J Am Coll Cardiol 2009; 54: 812-819.

32 Yoshikane Y, Yoshinaga M, Hamamoto K. Hirose S: A case of long QT syndrome with triple gene abnormalities: Digenic mutations in $\mathrm{KCNH} 2$ and SCN5A and gene variant in KCNE1. Heart Rhythm 2012; 10: 600-603.

33 Marjamaa A, Newton-Cheh C, Porthan $\mathrm{K}$ et al: Common candidate gene variants are associated with QT interval duration in the general population. J Intern Med 2009; 265: 448-458.

34 Rivero A, Curtis AB: Sex differences in arrhythmias. Curr Opin Cardiol 2010; 25 : $8-15$.

35 Schwartz PJ, Priori SG, Spazzolini C et al: Genotype-phenotype correlation in the long-QT syndrome: gene-specific triggers for life-threatening arrhythmias. Circulation 2001; 103: 89-95.

36 Frousios K, Iliopoulos CS, Schlitt T, Simpson MA: Predicting the functional consequences of non-synonymous DNA sequence variants-evaluation of bioinformatics tools and development of a consensus strategy. Genomics 2013; 102: 223-228

37 Giudicessi JR, Ackerman MJ: Genotype- and phenotype-guided management of congenital long QT syndrome. Curr Prob/ Cardiol 2013; 38: 417-455.

38 Giudicessi JR, Ackerman MJ: Determinants of incomplete penetrance and variable expressivity in heritable cardiac arrhythmia syndromes. Trans/ Res 2013; 161: 1-14.

39 Clancy CE, Kass RS: Defective cardiac ion channels: from mutations to clinical syndromes. J Clin Investig 2002; 110: 1075-1077.

Supplementary Information accompanies this paper on European Journal of Human Genetics website (http://www.nature.com/ejhg) 